\title{
Plastic deformations in the ring spherical shells
}

\author{
Avgustina Astakhova* \\ Moscow State University of Civil Engineering, Yaroslavskoe shosse, 26, Moscow, 129337, Russia
}

\begin{abstract}
In the present work the results of the study of plastic deformations distribution in the thickness in ring spherical shells are presented. Resolving differential equations system is based on the Hirchhoff-Lave hypothesis, linear thin shells theory and small elasticplastic deformations theory. The studying of the development area of plastic deformations in shells thickness are performed with using the results of the elastic solutions method. The basic relations of elastic solutions method that allow to determine the distribution areas of plastic deformations in shells thickness and along the generatrix are presented. The diagram of intense stress dependence from the strain intensity with linear hardening is received. The numerical solution is performed by orthogonal run method. Long and short spherical shells under the operation of three evenly distributed ring loads are observed. The shells have a tough jamming along the contour at the bottom and at the top. Dependency between tension intensity and deformations intensity is accepted for the case of a material linear hardening. Area of plastic deformations in shells thickness for three kinds of ring spherical shells are shown. The results for the loads differed by the value in twice are presented.
\end{abstract}

\section{State of the problem}

The studying of the stress-strain state of constructions is considered that physical nonlinearity of material is widely used in engineering practice. It is connected with the tendency to consider strength properties of material more thorough for providing the reliable work of the constructions under the loads.

In the present work the stress-strain state of thin isotropic spherical shells with constant thickness beyond the elastic limit under three evenly distributed loads along the parallel is studied. It is supposed that the values of loads such are that linear theory is used for determining the stress-strain state. It is correctly said that the Hirchhoff-Lave hypothesis and deformations are smaller than the shell thickness [1-2]. The shells may be long and short, withdrawn and circular, and the shells with different kinds of supports. The outer effect is set in the form of superposition of evenly distributed finite number along the parallel of loads operating along the rotation axis $\mathrm{Oz}(r, z$-cylindrical coordinates).

\footnotetext{
* Corresponding author: ast965@yandex.ru
} 


\section{The method of solution}

The medium surface of the shell is attributed to the curved orthogonal coordinate system $s$, $\theta, \zeta(\mathrm{s}-$ generatrix arc length, $\theta$ - angle in the parallel circle, $\zeta$ - normal to the surface) (fig.1).

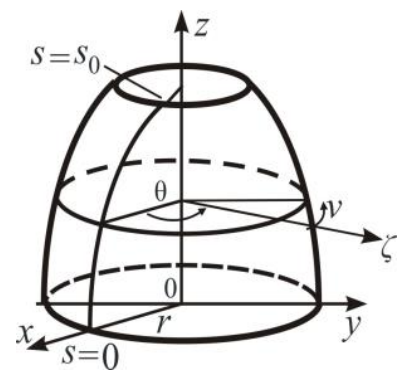

Fig. 1. Shell mid surface.

Resolving equations system is withdrawn from equilibrium equations, geometrical relations of linear theory presented in partial derivative, and physical relations of the theory of small elastic-plastic deformations [2-3] (in consideration the hypothesis of material incompressibility). Stress-strain state functions are decomposed to Fourier series along the circumferential coordinate $\theta$. Resolving system is a system of eight ordinary $1^{\text {st }}$ order differential equations with variable coefficients according to the coefficients of the unknown functions series $N\left(N_{r}, N_{z}, \bar{S}, M_{s}, u_{r}, u_{z}, v, \zeta_{s}\right)$ [4]. In the right part the system concludes the Fourier series coefficients of load elements and terms considering physical nonlinearity of material $\Delta(1)$ :

$$
N^{\prime}=A(s) N+\Delta
$$

Resolution functions are: $N_{r}$ - radial force, $N_{z}$ - force along the axis $z$ (axial force), $\hat{S}$ - generalized tangential stress, $M_{s}-$ meridional bending moment, $u_{r}-$ radial displacement, $u_{z}-$ axial displacement, $v-$ tangential displacement, $\zeta_{s}-$ section rotation angle angular deflection.

The boundary problem where eight boundary conditions set at the left (with $s=0$ ) and right $(s=s 0)$ ends of generatrix is solved. The resolving system integrates to the orthogonal run method [4-5].

The functions expressed physical nonlinearity of the problem are not equal to zero in the points of the formation of plastic deformations. The relations (2) - (6) allow to determine the areas of plastic deformations distribution along the shell thickness at each iteration $n(n$ $=1,2,3, \ldots)$ of the of the elastic solutions metod [3], [7-8].

$$
\begin{gathered}
J_{1 n}=\int_{-h / 2}^{h / 2} \psi_{n} d z, \quad J_{2 n}=\int_{-h / 2}^{h / 2} \psi_{n} z d z, \quad J_{3 n}=\int_{-h / 2}^{h / 2} \psi_{n} z^{2} d z, \\
\psi_{n}=\frac{\sigma_{i, n-1}}{\varepsilon_{i, n-1}}, \quad \varepsilon_{i, n-1}=\frac{2}{\sqrt{3}} \sqrt{P_{\varepsilon, n-1}+2 z P_{\varepsilon \kappa, n-1}+z^{2} P_{\kappa, n-1}}, \\
P_{\varepsilon, n-1}=\varepsilon_{1, n-1}^{2}+\varepsilon_{1, n-1} \varepsilon_{2, n-1}+\varepsilon_{2, n-1}^{2}+\frac{1}{4} \gamma_{12, n-1}^{2}, \\
P_{\varepsilon \kappa, n-1}=\varepsilon_{1, n-1} \kappa_{1, n-1}+\varepsilon_{2, n-1} \kappa_{2, n-1}+\frac{1}{2}\left(\varepsilon_{1, n-1} \kappa_{2, n-1}+\varepsilon_{2, n-1} \kappa_{1, n-1}\right)+
\end{gathered}
$$




$$
+\frac{1}{2} \gamma_{12, n-1} \kappa_{12, n-1}, \quad P_{\kappa, n-1}=\kappa_{1, n-1}^{2}+\kappa_{1, n-1} \kappa_{2, n-1}+\kappa_{2, n-1}^{2}+\kappa_{12, n-1}^{2}
$$

For example, the dependency between tension intensity and deformation intensity for the case of the linear hardness is expressed by the next linear functions (fig.2):

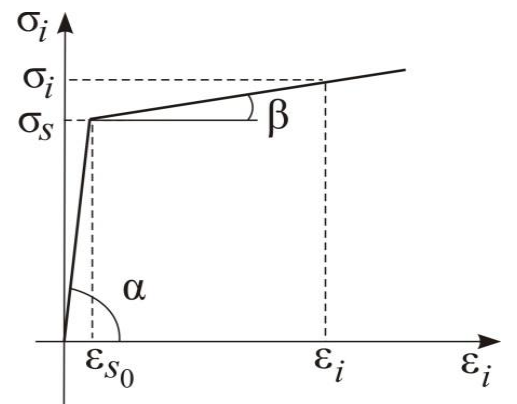

Fig. 2. Dependency between tension intensity and deformations intensity is accepted for the case of a material linear hardening.

Designated: $\sigma_{i}, \varepsilon_{i}$ - tension intensity value and deformations intensity in the random point, $\sigma_{\mathrm{s}}, \varepsilon_{s_{0}}-$ tension intensity and deformations intensity on the border between the elastic and plastic areas are noted (fig.2).

$$
\text { Where } \varepsilon_{i} \leq \varepsilon_{s_{0}} \sigma_{i}=E \varepsilon_{i}
$$

Where $\varepsilon_{i}>\varepsilon_{s_{0}} \sigma_{i}=E \varepsilon_{i}\left[1-\lambda\left(1-\frac{\varepsilon_{s_{0}}}{\varepsilon_{i}}\right)\right], \lambda=\frac{\operatorname{tg} \alpha-\operatorname{tg} \beta}{\operatorname{tg} \alpha}=\frac{E-E_{1}}{E}$

Let's note the minimum value of deformations intensity function $\varepsilon_{i}$ along the shell thickness $h-\varepsilon_{i_{0}}$, deformations intensity value on the inner surface $(z=-h / 2)-\varepsilon_{i_{1}}$, deformations intensity value on the outer surface $(z=h / 2)-\varepsilon_{i_{2}}$, the functions are determined by the next formulas:

$$
\begin{gathered}
\varepsilon_{i_{0}}=\frac{2}{\sqrt{3}} \sqrt{P_{\varepsilon}-\frac{P_{\varepsilon \kappa}^{2}}{P_{\kappa}}} \\
\varepsilon_{i_{1}}=\frac{2}{\sqrt{3}} \sqrt{P_{\varepsilon}-h P_{\varepsilon \kappa}+\frac{h^{2}}{4} P_{\kappa}} \text { with } z=-h / 2 \\
\varepsilon_{i_{2}}=\frac{2}{\sqrt{3}} \sqrt{P_{\varepsilon}+h P_{\varepsilon \kappa}+\frac{h^{2}}{4} P_{\kappa}} \text { with } z=h / 2
\end{gathered}
$$

If the minimum value of deformations intensity function $\varepsilon_{i_{0}}$ is located beyond the shell thickness $h$ at the distance $z_{0}$ of the medium surface where plastic deformations are located near outer or inter shell surface (fig. 3) or from the side of one of the surfaces, the value of toughness are determined by the next relations. 


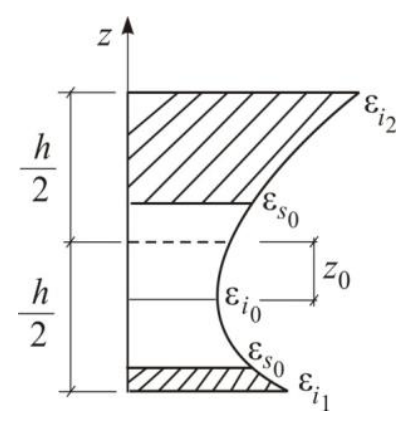

Fig.3. Plastic deformation area located near the inter and outer shell surfaces.

$$
\begin{gathered}
\text { при }-\frac{h}{2} \leq\left(z_{0}=-\frac{P_{\varepsilon \kappa}}{P_{\kappa}}\right) \leq \frac{h}{2} \\
J_{1}=\frac{\sqrt{3}}{2 \sqrt{P_{\kappa}}}, J_{2}=-\frac{\sqrt{3}}{2} \frac{P_{\varepsilon \kappa}}{P_{\kappa} \sqrt{P_{\kappa}}} B_{0}+\frac{3}{4 P_{\kappa}} A_{0}, \\
J_{3}=\frac{3 \sqrt{3}}{8 P_{\kappa} \sqrt{P_{\kappa}}} C_{0}+\frac{\sqrt{3}}{2} \frac{P_{\varepsilon \kappa}^{2}}{P_{\kappa}^{2} \sqrt{P_{\kappa}}} B_{0}-\frac{3 P_{\varepsilon \kappa}}{2 P_{\kappa}^{2}} A_{0}, \\
\text { где } A_{0}=\int_{\varepsilon_{i_{1}}}^{\varepsilon_{i_{2}}} \sigma_{i} d \varepsilon_{i}, B_{0}=\int_{\varepsilon_{i_{i 0}}}^{\varepsilon_{i_{1}}} \frac{\sigma_{i} d \varepsilon_{i}}{\sqrt{\varepsilon_{i}^{2}-\varepsilon_{i_{0}}^{2}}}+\int_{\varepsilon_{i_{0}}}^{\varepsilon_{i_{0}}} \frac{\sigma_{i} d \varepsilon_{i}}{\sqrt{\varepsilon_{i}^{2}-\varepsilon_{i_{0}}^{2}}}, \\
C_{0}=\int_{\varepsilon_{i_{0}}}^{\varepsilon_{\varepsilon_{1}}} \sigma_{i} \sqrt{\varepsilon_{i}^{2}-\varepsilon_{i_{0}}^{2}} d \varepsilon_{i}+\int_{\varepsilon_{i_{0}}}^{\varepsilon_{i_{2}}} \sigma_{i} \sqrt{\varepsilon_{i}^{2}-\varepsilon_{i_{0}}^{2}} d \varepsilon_{i}
\end{gathered}
$$

If the minimum value of deformations intensity function $\varepsilon_{i_{0}}$ is located beyond the shell thickness $h$ at the distance $z_{0}$ of medium surface (fig.4a, b) where plastic deformations are located near outer (fig.4a) or inter shell surface (fig.4b), the toughness is determined by the helping of coefficients $A_{1}, B_{1}, C_{1}$.

a)

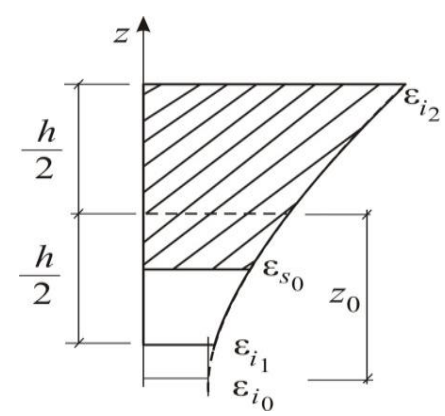

b)

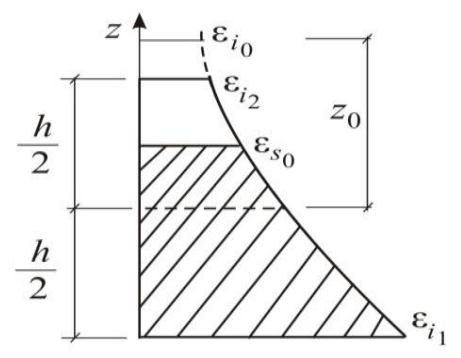

Fig.4. Plastic deformation areas with the minimum value of the deformations intensity functions $\varepsilon_{i_{0}}$ located beyond the shell thickness , a) plastic deformations are located from the side of the outer shell surface, b) plastic deformations are located from the side of the inner shell surface.

With $\left(z_{0}=-\frac{P_{\varepsilon \kappa}}{P_{\kappa}}\right)>\frac{h}{2}$ or $\left(z_{0}=-\frac{P_{\varepsilon \kappa}}{P_{\kappa}}\right)>-\frac{h}{2}$, the constants $A_{0}, B_{0}, C_{0}$ in the expressions (17) and (18) are replaced with $A_{1}, B_{1}, C_{1}$ : 


$$
\begin{array}{r}
A_{1}=A_{0}=\int_{\varepsilon_{i_{1}}}^{\varepsilon_{i_{2}}} \sigma_{i} d \varepsilon_{i} \\
B_{1}=\frac{\varepsilon_{i_{2}}-\varepsilon_{i_{1}}}{\left|\varepsilon_{i_{2}}-\varepsilon_{i_{1}}\right|} \int_{\varepsilon_{i_{1}}}^{\varepsilon_{i_{2}}} \frac{\sigma_{i} d \varepsilon_{i}}{\sqrt{\varepsilon_{i}^{2}-\varepsilon_{i_{0}}^{2}}}, C_{1}=\frac{\varepsilon_{i_{2}}-\varepsilon_{i_{1}}}{\left|\varepsilon_{i_{2}}-\varepsilon_{i_{1}}\right|} \int_{\varepsilon_{i_{1}}}^{\varepsilon_{i_{1}}} \sigma_{i} \sqrt{\varepsilon_{i}^{2}-\varepsilon_{i_{0}}^{2}} d \varepsilon_{i}
\end{array}
$$

\section{Results}

The stress-strain state in the ring spherical shells under the action of three evenly distributed ring loads applied to the generatrix $s$ arc with the step $0,25 \mathrm{~s}$ is examined (fig.5). The shells with the angle of half-life $\alpha=60^{\circ}, 90^{\circ}, 160^{\circ}$ and the angle of departure from rotation axis at the top $\delta=5^{\circ}$ are observed. The material is considered to be incompressible, Poisson coefficients are accepted to be equal to 0,5 in the elastic and elastic-plastic areas. The steel stretch diagram is approximated to polygonal line with hardening module $\lambda=$ 0,95 , the deformation intensity corresponded to the beginning of fluidity $\varepsilon_{S}=10^{-3}$, is taken.

For the shell with the relation of the radius to the thickness $\mathrm{R} / h=100$ with tough jamming along the top and bottom edge of the shell of the expansion area, plastic deformations are shown in the fig. 1 . In the short shells $\alpha=60^{\circ}$ and in the medium length shells $\alpha=90^{\circ}$ with the load $P / E h=0,143 \cdot 10^{-3}$ they are extended along the length $0,006 \mathrm{~s}$ on the outer and inner surfaces. In the long spherical shells $\alpha=160^{\circ}$ with the same value of the load the area of plastic deformations that embrace all the thickness of shell is extended along the length $0,24 \mathrm{~s}$ from the lower support and along the length $0,06 \mathrm{~s}$ between 1 st ring load and higher support. In the shells $\alpha=60^{\circ}$ and $\alpha=90^{\circ}$ plastic deformations along the thickness of generatrix at the length $0,14 \mathrm{~s}$ between 1 st ring load and higher support, the common area of their expansion compiles appropriately $0,44 s$ and $0,3 s$. In other part of generatrix for $\alpha=60^{\circ}$, plastic deformations are located near the outer and inner surfaces of the shell, with $\alpha=90^{\circ}$,encompass all the thickness along the length $0,12 \mathrm{~s}$ and $0,04 \mathrm{~s}$ (fig.6). The area of plastic deformations with $\alpha=160^{\circ}$ is extended along the generatrix from higher to lower support at 0,32 s (fig.7).

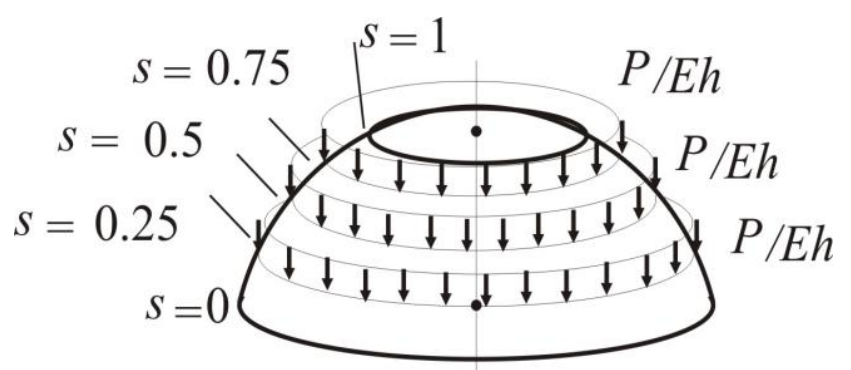

Fig. 5. Ring spherical shell under the action of three evenly distributed ring loads. 

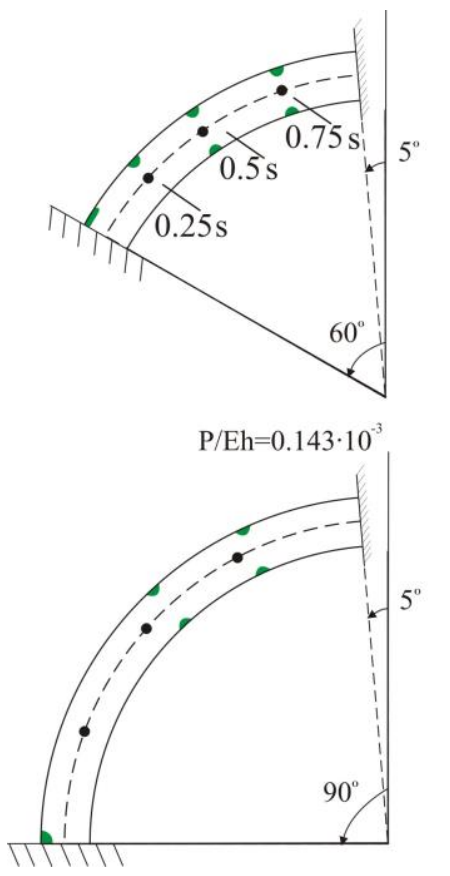

$\mathrm{R} / \mathrm{h}=100$
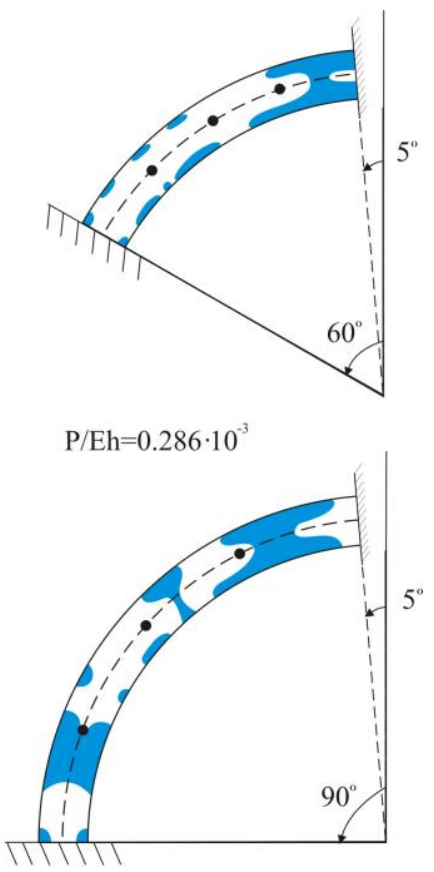

Fig. 6. Short and average shells with rigid attachment along of the top and of the bottom contours.
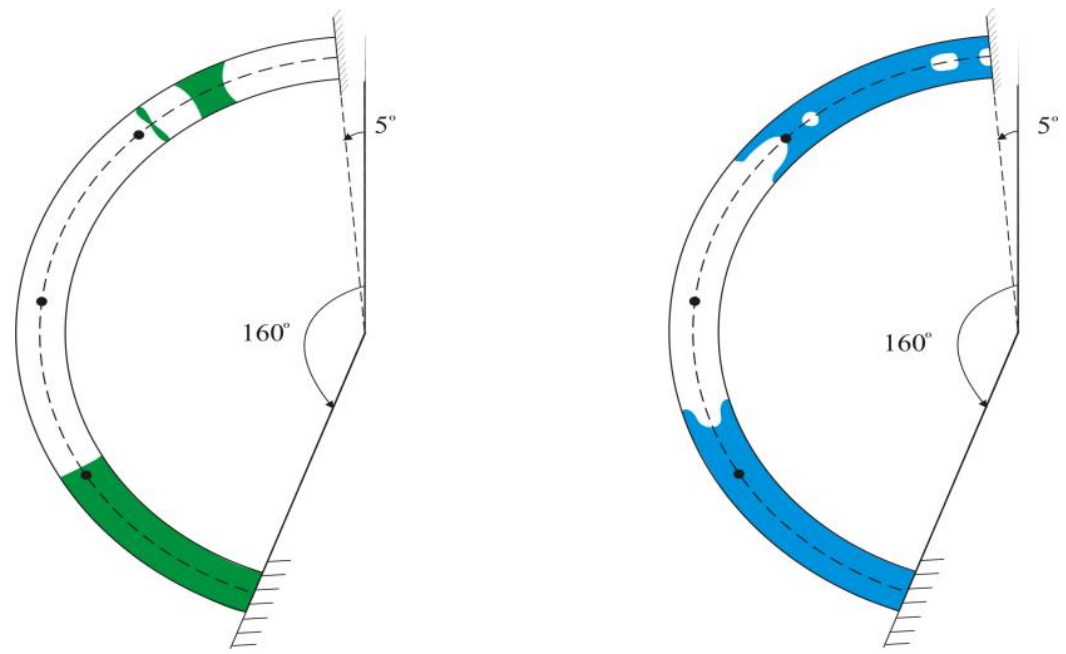

Fig. 7. Long shells with rigid attachment along of the top and of the bottom contours.

\section{Conclusions}

The increase of the load in twice essentially influences on the expansion of plastic deformations as along the thickness of shell as along the generatrix.

Meridian curved moments in the short shells and in the medium length shells take the maximum values near the ring loads applications, in the long shells maximum values 
appear in the tough jamming area and exceed the value in other points roughly in twice or in three times.

The components of the horizontal displacements of the points of the middle surface of the shell are approximately an order of magnitude larger than vertical components of the displacement. They take maximum values near the lower support.

\section{References}

1. V. Vlasov, Shell theory and its application to the technics. 1, 528, (1962)

2. A. Ilyushin, Plasticity, 2, 3,9 Mb, (2004)

3. I. Tsurkov, Col. papers of MISI, Towa, 47, 12 (1965)

4. Y. Grigorenko, F.T. Vasilenko, J. Appl. M., 3, 6 (1968)

5. A. Astakhova, J. M. of Rig. B, 4, 6 (1985)

6. A. Astakhova, Matec. Web Conf., 86 (2016)

7. A. Astakhova, "NAUKOVEDENIE", online mag. 5, 9, 12 (2017)

8. A.Y. Astakhova, Matec. Web Conf., 196 (2018) 